\title{
Efficacy of the Resistive Index of Color Doppler Ultrasonography in Differential Diagnosis of Benign and Malignant Hepatic Space Occupying Lesions
}

\author{
Shimu $\mathrm{F}^{1}$, Karim E $\mathrm{E}^{2}$, Khan $\mathrm{AH}^{3}$, Ahmed A ${ }^{4}$, Parvin $\mathrm{S}^{5}$, Rafat $\mathrm{S}^{6}$, Akther $\mathrm{SMQ}^{7}$
}

\begin{abstract}
Background: Write the background in 1 to 2 sentences]. Objective: The purpose of the present study was to estimate the efficacy of resistive index (RI) of color Doppler ultrasonography in different space occupying liver lesions. Methodology: This cross sectional study was conducted at in Bangabandhu Sheik Mujib Medical University (BSMMU), Dhaka from July 2010 to December 2012. All the patients presented with the suspicion of hepatic space occupying lesions were enrolled for this study who were later diagnosed clinically or ultrasonographically. This patients were also examined by color Doppler flow imaging. All patients were examined by gray scale Ultrasonography, color Doppler and FNAC. To visualize the blood flow, standard color Doppler sonography is used for each lesion. Within the lesions, pulsed Doppler samples are assessed whenever possible on the basis of pulsatile flow. At least three measurements of resistive index (RI) of intra tumoral and peritumoral arterial blood flow would be the last mean value. Results: The detection rate of arterial flow in primary malignant tumors was $94.4 \%$ and $87.7 \%$ in hepatic metastasis. Doppler spectrum analysis showed the resistance index in primary malignant tumor was $0.75 \pm 0.12,0.73 \pm 0.09$ in metastatic tumor and was below 0.6 in benign lesions. The difference was significant $(\mathrm{p}<0.001)$. This difference was related with its histopathologic structure. The arterial flow with RI $>0.6$ in CDFI within the liver lesion can be regarded as a criterion of malignant tumors, RI $<0.6$ can be regarded as benign lesions. Conclusion: RI is more helpful in differential diagnosis of benign and malignant lever lesions. [J Shaheed Suhrawardy Med Coll, 2014;6(1):27-30]
\end{abstract}

Keywords: Color Doppler, ultrasonography, resistive index, hepatic space occupying lesions

Received: March 2014; Revised: April 2014; Accepted: May 2014

\section{Introduction}

Hepatic lesions are a common problem referred to radiologists for evaluation ${ }^{1-2}$. Grey scale ultrasonography and CT scan is being used for the diagnosis and staging of different hepatic lesions ${ }^{3-5}$. In tandem with advances in grey scale ultrasound, color Doppler has tremendous forcemultiplier effect in detection hepatic diseases especially those of haemodynamic in nature, obviating the need for invasive procedures with their attendant risk of morbidity and mortality $^{6}$. Resistive index (RI) is one of the indices of measurement of the shape of flow waveform ${ }^{4}$. RI is based on the maximum Doppler shift waveform.

Benign and malignant lesions vary in their appearance and resistive index in Doppler ${ }^{4}$. Primary liver lesion differs from the vascularity of the hepatic secondaries, and able to discriminate these two without need of surgical intervention. Therefore, colour Doppler ultrasonography is a good option for the radiological diagnosis of Hepatocellelar Carcirome (HCC) hepatic secondaries and also to differentiate benign and malignant lesions. The purpose of the present study was to estimate the efficacy of resistive index (RI) of color Doppler ultrasonography in different space occupying lesions of liver.

\section{Methodology}

This study was designed as cross sectional study which was conducted Department of Radiology \& Imaging, Bangabandhu Sheik

1. Dr. Farhana Shimu, Assistant Professor, Department of Radiology \& Imaging, Dhaka Central International Medical College, Dhaka

2. Prof. Dr. Enayet Karim, Professor, Department of Radiology \& Imaging, Bangabandhu Sheik Mujib Medical University, Dhaka

3. Dr. Abul Hashem Khan, Assistant Professor, Department of Surgery, Sir Salimullah Medical College, Dhaka

4. Dr. Akhter Ahmed, Assistant Professor, Department of Surgery, Shaheed Suhrawardy Medical College, Dhaka

5. Major Dr. Sultana Parvin, Consultant, Department of Radiology \& Imaging, Armed Forces Medical College, Dhaka

6. Dr. Sania Rafat, Junior Consultant, Department of Radiology \& Imaging, Delta Medical College \& Hospital, Dhaka

7. Dr. S.M. Quamrul Akther, Assistant Professor, Department of Surgery, Shaheed Suhrawardy Medical College, Dhaka

\section{Correspondence}

Dr. Farhana Shimu, Assistant Professor, Department of Radiology \& Imaging, Dhaka Central International Medical College, Ring Road, Shamoly, Dhaka-1207, Bangladesh; Email: shimu.farhana35@gmail.com; Cell no.: +8801711160152

Conflict of interest: None

Financial Support: None

Contributions by authors: FS, EK, AHK \& AA involved in protocol prepation, data management \& manuscript writing; SP,SR \& SMQA contributed to revision of paper. 
Mujib Medical University, Dhaka from July 2010 to December 2012 for period of two and half years. At first all the patients were evaluated by detailed history and clinical examination. All patients were examined by gray scale Ultrasonography, color Doppler and FNAC. The cytopathology reports were collected and correlated with RI findings at color Doppler. After informing all the necessary information to the patients regarding the research study, data were collected in a pre-designed structured questionnaire. All patients were undergoing grey scale USG and Doppler USG in the morning after overnight fast. The color Doppler unit (GE Voluson 730 PRO) and a 3.5-5 MHz phased array linear electronic probe were used. To visualize the blood flow, standard color Doppler sonography is used for each lesion, and pulsed Doppler was optimized to detect the low flow components by diminishing pulse repetition frequencies, usually down to $500 \mathrm{~Hz}$ and adapting frequency filters. The color gain was manipulated until noise began to exceed the homogeneous single color background of color Doppler scans. Within the lesions, pulsed Doppler samples are assessed whenever possible on the basis of pulsatile flow2. At least three measurements of resistive index (RI) of intra tumoral and peritumoral arterial blood flow would be the last mean value. Only those showing the highest SPV values obtained with pulsed Doppler were taken into account. For statistical analysis, one way ANOVA and student $t$ test were performed.

\section{Results}

175 patients

Maximum patients were in the age group above 60 years (31.4\%). Patients of age group 50-60 were 54(30.8\%) in number. Youngest age group was less than 30 years. Their number was $6(3.4 \%)$ in total. In this series, most hepatic space occupying lesions were found in male. There were $140(80 \%)$ male patients in contrast to $35(20 \%)$ female patients. Patients were suffering from weakness $(80 \%), 122$ patients had weight loss, 78 patients complained of abdominal pain, 56 had palpable mass in abdomen. About $70 \%$ patients had weight loss, $80 \%$ had weight loss and $44.5 \%$ had abdominal pain. HCC was the most frequently diagnosed by arterial flow rate. Benign lesions like abscess and cirrhotic nodules didn't show arterial flow frequently. Only $27.3 \%$ abscess showed arterial flow in and around tumor, whereas only $18.2 \%$ cirrhotic nodule showed flow. Most tumors are primary malignant (120). Among primary lesions, 40 lesions were less than $30 \mathrm{~mm}, 54$ are between 30 $60 \mathrm{~mm}$, and 26 cases are over $60 \mathrm{~mm}$. Benign cases are 48 in number. Most benign cases (21) are less than $30 \mathrm{~mm}$ in size, 18 are between $30-60 \mathrm{~mm}$, and 9 cases are over $60 \mathrm{~mm} .120$ cases ( $68.5 \%$ ) were diagnosed by RI as HCC. But in FNAC, $118(67.42 \%)$ were cytologically positive. In hepatic metastasis, 7 cases (4\%) were diagnosed by RI in color Doppler. Out of that 7 cases, all 7 (4\%) cases were diagnosed same by FNAC. In cases of benign lesions, 48 were diagnosed by RI. FNAC positive cases are 50. Malignant lesins showing RI over 0.7 and benign below 0.6. MeanRI in
HCC is 0.75 , in hepatic secondaries 0.73 and in focal noduler hyperplasia RI mean is 0.58 . RI value in $<30 \mathrm{~mm}$ lesions are about 0.77 , in $30-60 \mathrm{~mm}$ lesions, RI is about 0.76 , and $\mathrm{RI}$ is 0.71 in lesions more than $60 \mathrm{~mm}$. RI positive benign lesions were 48 . Among them, only 28 lesions were proved benign in FNAC, other 20 were proved malignant. RI negative cases were 127 , among them 22 cases were diagnosed as benign in FNAC, and 105 cases were diagnosed not benign. RI is not very useful in accuracy in diagnosing benign hepatic lesions. Sensitivity of benign lesions are $56 \%$, specificity is $84 \%$, positive predictive value is $58.3 \%$, negative predictive value is $82.6 \%$, and accuracy is $76 \%$. Total 127 cases were diagnosed as malignant in RI. Among them, 118 cases were confirmed by FNAC, but 9 cases were diagnosed as benign in FNAC. 7 cases were diagnosed as benign in RI but became positive in FNAC. Total 50 cases were diagnosed as benign in FNAC, where as 48 in RI. Malignant lesions are more sensitive in RI. RI sensitivity is $94.4 \%$, Specificity is $82 \%$, PPV is $92.9 \%$, NPV is $85.4 \%$, and accuracy is $90.8 \%$. RI diagnosed 120 cases as HCC. Among those, 6 cases were diagnosed as not HCC and 114 as HCC in FNAC examination. 51 cases were diagnosed as not $\mathrm{HCC}$ in both examinations. 4 cases were diagnosed as HCC by Cytopathology, but not HCC in RI. Malignant lesions are more sensitive in RI. RI sensitivity is $96.6 \%$, Specificity is $89.4 \%$, PPV is $95 \%$, NPV is 92.7 , and accuracy is $94.2 \%$. 7 cases were diagnosed hepatic secondaries by RI. Among them one case was diagnosed as not secondary. 168 cases were diagnosed as not secondary by RI. Among those, only one case was diagnosed as secondary in FNAC. In hepatic metastasis cases, RI sensitivity, specificity, PPV, NPV and accuracy is $85.7 \%, 99.4 \%, 85.7 \%, 99.4 \%$ and $98.8 \%$ respectively.

\section{Discussion}

Maximum patients are in the age group above $60(31.4 \%)$ years. Patients of age group 50-60 years were 54(30.8\%) in number. Youngest age group was less than 30 years. Their number was 6(3.4\%) in total. In another study the mean age of patients having space occupying liver lesion was 58 years with age range from 40 to 80 years ${ }^{7}$. Further more mean age of the patients having liver mass was 65 years with age ranged from 46 to 88 years $^{8}$, which are higher than the present study. This may be due to increased life expectancy in their respective country population.

In this series, most hepatic space occupying lesions were found in male. There were 140 male patients $(80 \%)$ in contrast to 35 female patients (20\%). In as study found male female ratio 2.5:1. Semelka et al male to female ratio 2.4:1, which do not match with study. This may indicate regional variation in disease process.

Weakness, weight loss and abdominal pain are shown as main clinical picture of hepatic mass. Most patients complained of weakness $(80 \%)$, weight loss $(69.7 \%)$, abdominal pain $(44.5 \%)$ and palpable abdominal mass $(32 \%)$. Only 4 patients presented with jaundice. There is difference in detection rates of arterial blood flow in different hepatic 
space occupying lesions. $\mathrm{HCC}$ is most frequently diagnosed. Benign lesions like abscess and cirrhotic nodules do not show arterial flow frequently. Only $27.3 \%$ abscess show arterial flow in and around tumor, where as only $18.2 \%$ cirrhotic nodule show flow.

In this study, most tumors are primary malignant (120). Among primary lesions, 40 lesions were less than $30 \mathrm{~mm}, 54$ are between $30-60 \mathrm{~mm}$, and 26 cases are over $60 \mathrm{~mm}$. Benign cases are 48 in number. Most benign cases (21) are less than $30 \mathrm{~mm}$ in size, 18 are between $30-60 \mathrm{~mm}$, and 9 cases are over $60 \mathrm{~mm}$. So, benign cases were mostly small in size in contrast to malignant lesions.

Here, 120 cases $(68.5 \%)$ were diagnosed by RI as HCC. But in FNAC, $118(67.42 \%)$ were cytologically positive. In hepatic metastasis, 7(4\%) cases were diagnosed by RI in color Doppler. Out of that 7 cases, all $7(100 \%)$ cases were diagnosed similar diagnosis by FNAC. In cases of benign lesions, 48 cases were diagnosed by RI. FNAC positive cases are 50 . RI values differ in different hepatic space occupying lesions. Malignant lesions showing RI over 0.7 and benign below 0.6. Mean RI in HCC is 0.75 , in hepatic secondaries 0.73 and in focal noduler hyperplasia RI mean is 0.58 . This series showed that, more than $60 \mathrm{~mm}$ lesions are diagnosed $97 \%, 30-60 \mathrm{~mm}$ lesions are diagnosed by $93.3 \%$, and smallest lesions $(<30 \mathrm{~mm})$ are diagnosed $87.9 \%$. RI value in $<30 \mathrm{~mm}$ lesions are about 0.77 , in $30-60 \mathrm{~mm}$ lesions, RI is about 0.76 , and RI is 0.71 in lesions more than $60 \mathrm{~mm}$.

In this series, RI positive benign lesions were 48. Among them, only 28 lesions were proved benign in FNAC, other 20 were proved malignant. RI negative cases were 127, among them 22 cases was diagnosed as benign in FNAC, and 105 cases were diagnosed not benign. Here, total 127 cases were diagnosed as malignant in RI. Among them, 118 cases were confirmed by FNAC, but 9 cases were diagnosed as benign in FNAC. 7 cases were diagnosed as benign in RI but became positive in FNAC. Total 50 cases were diagnosed as benign in FNAC, where as 48 in RI.

Type of signals (arterial or venous) and its distribution detected by color and pulsed Doppler was more helpful than the assessment of quantitative spectral parameters obtained by pulsed Doppler ${ }^{9}$. Some researchers ${ }^{10-13}$ attempted to characterize tumors by quantitative spectral criteria only, and found that systolic peak velocity (SPV) was above $70 \mathrm{~cm} / \mathrm{s}$ in 32 hepatomas in their series, the velocities differed significantly from those found in metastases and hemangiomas. The mean of SPV in hepatomas was significantly higher than that of metastases, and hemangiomas ${ }^{14}$. The arteries supplying HCCs had lower PI and higher $\mathrm{PSV}^{3}$. Some investigators ${ }^{9}$ found a remarkable overlap between the spectral values of metastases and primary carcinomas. The velocity and RI had no significance in differential diagnosis of tumors ${ }^{10}$. The value of SPV could usually be affected by the Doppler anglel; however, in most cases, the course detection rate of tumor vessels could not be determined. Therefore, the mean of SPV is varied greatly and high SPV is usually presented in larger tumors, and is not significant in the differentiation of small lesions. RI (Resistive index $=(\mathrm{Vsp}-\mathrm{Ved}) / \mathrm{Vsp}$ ) presents the Resistive of distal vessels. It is not influenced by the Doppler angle. $\mathrm{RI}>0.5$ was usually observed in malignant tumors, and $\mathrm{RI}<0.5$ was found in the hemangiomas. In our further study, the statistical analysis of 175 patients also showed that RI in malignant tumors was significantly higher than that in benign tumors $^{16}$. The average value of RI in primary liver malignant tumors was $0.75 \pm 0.12$, and $0.73 \pm 0.09$ in metastatic tumors. They were much higher than that of benign. Pulsed Doppler spectrum analysis showed that the lesions without any signal during diastole or with diastolic reversal spectrum were all malignant. The mean value of RI in hemangiomas was $0.55 \pm 0.08$. Some angioarchitectural features may also contribute to the explanation of the peculiar hemodynamic pattern observed. The nodule comprises exclusively arterial tumoral vessels, and hepatocytes are arranged in trabeculae of varying thickness that may compress the interposed vascular space, producing multiple "stenoses". Furthermore, the presence of pesudocapsules and cirrhotic parenchymas that usually surround HCCs might affect the venous outflow by compression of peritumoral portal branches. But in larger tumors (diameter $>6 \mathrm{~cm}$ ) with formation of $\mathrm{A}-\mathrm{V}$ shunt and destruction of pseudocapsules, the value of RI tended to be lower. In this series, combination of color Doppler flow imaging and RI was more helpful in differentiating malignant from benign tumors. Some of FNH, AML usually had more arterial flow just like malignant tumors, but they showed peculiar angioarchitectural features. FNH was characterized pathologically by cholangiolar proliferation associated with hyperplastic hepatocytes, blood vessels and fibrosis. AML was characterized pathologically by vessels with a thick muscle layer, showing more arterial flow signals like "blood ball". They often showed high peak velocity and low impedance, RI was usually $<0.6$. In the other aspect, RI was more useful to differentiate some benign lesions with less blood flow such as inflammatory pseudotumors of liver and cirrhotic nodules ${ }^{7}$. Inflammatory pseudotumors of liver had no blood flow or less peritumoral flow signals with $\mathrm{RI}<0.6$. Cirrhotic nodules usually had venous intratumoral flow or less peritumoral flow with $\mathrm{RI}<0.6$.

The factors affecting RI in the diagnosis of liver lesions include $^{10}$ sensitivity of the equipment; the difference in findings of flow is presumably due to the sensitivity of the equipment used for Doppler frequency shifts. Thus even lower and finer blood flows can probably be visualized if newer instruments are developed; management of the equipment is needed to visualize blood flow and pulsed Doppler is optimized to detect even low flow components by diminishing pulse repetition frequencies, usually down to $500 \mathrm{~Hz}$ and adapting frequency filters; color imaging of blood flow is insufficient when the tumor is located deep within in the liver; It is difficult to visualize when tumor size is less than $2 \mathrm{~cm}$ with deep location or near the diaphragm; cirrhotic parenchyma of the liver usually causes acoustic attenuation; the angle of Doppler can affect the sensitivity of CDFI.

It should be noticed that the lesion without blood flow signals is presumably due to the angio architectural features or the above factors. In this study, most of malignant tumors without blood flow are usually smaller than $6 \mathrm{~cm}$, and deeply located in the right posterior lobe of the liver or near the diaphragm, or located in the left lateral lobe with interference 
of the lung gas and the heart beating.

In this study, malignant lesions are proved most effective in diagnosis by RI. The sensitivity is $94.4 \%$, specificity is $82 \%$, PPV is $92.9 \%$, NPV is $85.4 \%$ and overall accuracy is $90.8 \%$. Among malignancy, primary liver cancer (HCC) is even better diagnosed by RI. In case of HCC, RI sensitivity is $96.6 \%$, specificity is $89.4 \%$, PPV is $95 \%$, NPV is $92.7 \%$ and overall accuracy is $94.2 \%$. For secondary metastasis, sensitivity is $85.7 \%$, specificity is $99.4 \%$, PPV is $85.7 \%$, NPV is $99.4 \%$ and overall accuracy is $98.8 \%$. In this comparison, benign lesions are less likely diagnosed by only RI. Here sensitivity is $56 \%$, specificity is $84 \%$, PPV is $58.3 \%$, NPV is $82.6 \%$ and overall accuracy is $76 \%$.

Data also shows that, larger the lesion easier to diagnose by RI. About $97 \%$ cases were diagnosed by RI, where tumor size was larger than $60 \mathrm{~nm}$. The mean RI was 0.75 in primary liver malignancy, 0.73 in hepatic secondaries, and less than 0.60 in all benign lesions. When intra-and/or peritumoral arterial blood flow is found, $\mathrm{RI}<0.6$ would strongly suggest a benign tumor. Simultaneous occurrence of both intra-and peritumoral arterial flow and RI.>.6 would strongly suggest malignancies. So that combined studies of the type of intra-and peritumoral flow signals in CDFI and the parameters of RI would be more helpful in differential diagnosis of benign and malignant liver tumors. Although sample size was calculated statistically, the original sample size was small in relation to huge number of population. True picture of the country cannot be achieved from this.

\section{Conclusion}

In conclusion, the type of flow signals (arterial and/or venous) and its distribution in CDFI and pulsed Doppler are helpful in differentiating benign from malignant lesions. The presence of intratumoral venous flow is strongly suggestive of benign tumors. Nationwide data collections from different corners of Bangladesh should be carried out.

\section{References}

1. Bolondi L, Gaiani S, Celli N, et al. Characterization of small nodules in cirrhosis by assessment of vascularity: the problem of hypovascular hepatocellular carcinoma. Hepatology 2005; 42: 27-34.

2. Bruix J, Sherman M; Practice Guidelines Committee, American Association for the Study of Liver Diseases. Management of hepatocellular carcinoma. Hepatology 2005; 42: 1208-1236.

3. Chen RC, Wang CK, Wang CS, et al. Depiction of vasculature in small hepatocellular carcinoma, and dysplastic nodules evaluated with carbon dioxide ultrasonography and angiography. Acta Radiol 2002; 43: 66-70.

4. Colombo M: Natural history of hepatocellular carcinoma. Ann Ital Chir 2008; 79(2): 91-7

5. Edmondson HA, Steiner PE. Primary carcinoma of the liver: a study of 100 cases among 48,900 necropsies. Cancer 1954; 7: 462-503.

6. Shaheen F, Gojwari T, Singh M, Rasool R, Banday HH. Color Doppler of abnormal liver circulation. JK Practitioner 2006; 13(4): 183-5

7. Gheorghe L, Iacob S, Gheorghe C. Real-time sonoelastography - a new application in the field of liver disease. J Gastrointestin Liver Dis 2008; 17 : $469-474$

8. Gish RG. Hepatocellular carcinoma: overcoming challenges in disease management. Clin Gastroenterol Hepatol 2006; 4: 252-261.

9. Herold C, Reck T, Fischler P, et al. Prognosis of a large cohort of patients with hepatocellular carcinoma in a single European centre. Liver 2002; 22: 23-28

10. Ikeda K, Saitoh S, Koida I, et al. Diagnosis and follow-up of small hepatocellular carcinoma with selective intra arterial digital subtraction angiography. Hepatology 1993;17: 1003-1007.

11. Kato K, Sugimoto H, Kanazumi N, Nomoto S, Takeda S, Nakao A. Intraoperative application of real-time tissue elastography for the diagnosis of liver tumors. Liver Int 2008; 28: 1264-1271

12. Koito K, Namieno T, Morita K. Differential diagnosis of small hepatocellular carcinoma and adenomatous hyperplasia with power Doppler sonography. AJR Am J Roentgenol 1998; 170: 157-161.

13. Kojiro M. Focus on dysplastic nodules and early hepatocellular carcinoma: an Eastern point of view. Liver Transpl 2004; 10(Suppl 1): S3-S8

14. Kubota K, Hisa N, Fujiwara Y, Fukumoto M, Yoshida D, Yoshida S. Evaluation of the intratumoral vasculature of hepatocellular carcinoma by power Doppler sonography: advantages and disadvantages versus conventional color Doppler sonography. Abdomen Imaging 2000; 25: 172-178

15. Llovet JM. Updated treatment approach to hepatocellular carcinoma. J Gastroenterol 2005; 40 225-235.

16. Munoz N, Bosch X. Epidemiology of hepatocellular carcinoma. In: Okuda K, Ishak KG, eds. Neoplasms of the liver. Tokyo: Springer, 1987:3-19 